\title{
A Redefinition of Somatosensory Areas in the Lateral Sulcus of Macaque Monkeys
}

\author{
Leah Krubitzer, Janine Clarey, Rowan Tweedale, Guy Elston, and Mike Calford \\ Vision, Touch and Hearing Research Centre, Department of Physiology and Pharmacology, University of \\ Queensland, Australia, 4072
}

\begin{abstract}
The present investigation was designed to determine the organization of somatosensory fields in the lateral sulcus of macaque monkeys using standard microelectrode recording techniques. Our results provide evidence for two complete representations of the body surface. We term these fields the second somatosensory area (SII) and the parietal ventral area (PV) because of their similarities in position, internal organization, and relationship to anterior parietal fields, as described for SII and PV in other mammals. Areas SII and PV are mirror-symmetrical representations of the body surface, sharing a common boundary at the representations of the digits of the hand and foot, lips, and mouth. These fields are located adjacent to the face representations of anterior parietal fields (areas $3 b, 1$, and 2), and are bounded ventrally and caudally by other regions of cortex in which neurons are responsive to somatic or multimodal stimulation. The finding of a double representation of the body surface in the region of cortex traditionally designated as SII may explain conflicting descriptions of SII organization in macaque monkeys. In addition, the present study raises some questions regarding the designation of serial processing pathways in Old World monkeys, by suggesting that fields may have been confused in studies demonstrating such pathways. We propose that SII and PV are components of a common plan of organization, and are present in many eutherian mammals.

[Key words: second somatosensory area, parietal ventral area, primates, somatosensory, lateral sulcus, serial processing, evolution]
\end{abstract}

For some time, anterior parietal cortex in primates was considered to contain a single, topographically organized representation of the body surface termed the primary somatosensory area (SI). However, modern microelectrode mapping studies in both primate (e.g., Merzenich et al., 1978; Kaas et al., 1979; Nelson et al., 1980; Sur et al., 1982; Pons et al., 1985), and nonprimate mammals (e.g., Darian-Smith et al., 1966; Johnson et al., 1982;

\footnotetext{
Received June 29, 1994; revised Oct. 19, 1994; accepted Dec. 7, 1994.

We thank Sherrie Florence, Marcello Rosa, and Tim Pons for their careful reading and helpful comments on this manuscript. We are also grateful to the staff of the Division of Ophthalmology, Department of Surgery, Princess Alexandra Hospital, and the John Curtin School of Medical Research for providing the monkeys. This work was supported by the Australian Research Council (ARC), the National Health and Medical Research Council (NHMRC), and a Special Research Centre Grant of the ARC.

Correspondence should be addressed to Leah Krubitzer, Center for Neuroscience, University of California, Davis, 1544 Newton Court, Davis, CA 95616.

Copyright $@ 1995$ Society for Neuroscience $\quad 0270-6474 / 95 / 153821-19 \$ 05.00 / 0$
}

Feldman and Johnson, 1988; Krubitzer and Calford, 1992; LeClerc et al., 1993) have demonstrated that this region of cortex consists of multiple, somatotopically organized representations. In macaque monkeys, it is well established that there are four anterior parietal fields: areas 1 and $3 \mathrm{~b}$ are mirror image representations of the cutaneous body surface (Kaas et al., 1979; Nelson et al., 1980), area 2 is a topographic representation of the deep receptors, and some cutaneous receptors (Pons et al., 1985), and area $3 \mathrm{a}$ is another deep representation, primarily of the muscle spindles (Jones and Porter, 1980; Wicsendanger and Milcs, 1982; Kaas and Pons, 1985, for review)

Until recently, it was also believed that lateral parietal cortex contained only a single field, the second somatosensory area (SII). However, electrophysiological mapping studies in a number of nonprimate species have demonstrated that the lateral somatosensory area is divisible into at least two representations: SII, located caudolaterally, and the parietal ventral area (PV) located rostrolaterally, in squirrels and flying foxes (Krubitzer et al., 1986; Krubitzer and Calford, 1992), and SII and the fourth somatosensory area (SIV) in cats (Clemo and Stein, 1982, 1983). Although there have been a number of investigations in the lateral sulcus region in primates, only in two New World monkeys have the boundaries of SII been delineated and additional representations identified. In the marmoset, double, mirror symmetrical body representations have been found in the region of cortex traditionally defined as SII (Krubitzer and Kaas, 1990), while in owl monkeys (Cusick et al., 1989), SII and a more ventral somatosensory area termed VS have been identified. The organization and connections of the two fields in the marmoset are similar to those described for SII and PV in squirrels and flying foxes. A lateral field in a similar position to VS in owl monkeys has also been recognized in the flying fox (Krubitzer and Calford, 1992).

In Old World monkeys, the few studies that have investigated the lateral sulcus, although well executed, have not used techniques that were ideal for examining its organization. For example, in studies in which single unit recordings were used to determine the topography of SII (e.g., Whitsel et al., 1969; Robinson and Burton, 1980) a limited region of cortex in any given animal was surveyed, and the topography of SII was derived by collapsing data across animals. Furthermore, in one of these studies, the resulting description of SII was limited only to the medial-to-lateral organization (Whitsel et al., 1969). Some of these earlier studies in macaque monkeys (Robinson and Burton, 1980; Burton and Robinson, 1981) have divided the lateral sulcus into a number of separate subdivisions, but these were based primarily on architectonic criteria with few recordings in any individual animal. 




Figure I. The organization of SII in macaque monkeys adapted from illustrations by Robinson and Burton $(1980 ; A)$. Pons et al. $(1988 ; B)$, and Friedman et al. $(1980 ; C)$. The studies in $A$ and $B$ utilized electrophysiological recording techniques to define the topographic organization of SII, while the study in $C$ used anatomical connections from anterior parietal fields. In the Robinson and Burton study $(A)$, SII comprises a very large region of cortex and spreads onto the insula of the lateral sulcus; there were two, spatially separate representations of the foot, forelimb, hindlimb, and maxillary and mandibular regions. $c c$, Caudal complex; $f$, facial; man, mandibular; max, maxillary; $r c$, rostral complex; to, tongue. Other abbreviations in 'lable 1.

In other studies in macaque monkeys, the connections of lateral somatosensory fields with anterior parietal fields (Friedman et al., 1980; Alloway et al., 1990) were used to determine the topographic organization of the SII region. However, this approach is limited in its precision since connections can only provide a general indication of topographic organization, and not all connections are to homotopic locations (e.g., Krubitzer et al., 1993). One study designed to reveal changes in SII after lesions in anterior parietal cortex (Pons et al., 1988), produced a detailed map of "SII," but the surrounding cortex was not designated. It is unclear whether this cortex was unmapped or unresponsive. Not surprisingly, examination of the results of these studies reveals a conflicting story (Fig. 1) with clear differences in the size, position, and somatotopy of the area termed "SII" by different investigators. We reasoned that lateral somatosensory cortex in Old World monkeys, as in other mammals, may contain several separate subdivisions, rather than one, very large area. Here we describe the somatotopic and architectonic organization of this region in Macaca fascicularis, and confirm our hypothesis that this region can be divided into at least two fields containing independent representations of the body surface.

\begin{tabular}{|c|c|}
\hline \multicolumn{2}{|c|}{ Cortical fields } \\
\hline A & auditory area \\
\hline PV & parietal ventral area \\
\hline SI & primary somatosensory area \\
\hline SII & second somatosensory are \\
\hline VS & ventral somatosensory area \\
\hline 1 & caudal somatosensory field (cutaneous) \\
\hline 2 & far caudal somatosensory field (deep) \\
\hline $3 a$ & far rostral somatosensory field (deep) \\
\hline $3 b$ & primary somatosensory area (SI), cutaneous \\
\hline 5 & posterior parietal area \\
\hline $7 \mathrm{~b}$ & posterior parietal area \\
\hline \multicolumn{2}{|l|}{ Sulci } \\
\hline $\mathrm{cs}$ & central sulcus \\
\hline ips & intraparietal sulcus \\
\hline lbls & lower bank of lateral sulcus \\
\hline ls & lateral sulcus \\
\hline ubls & upper bank of lateral sulcus \\
\hline \multicolumn{2}{|c|}{ Body parts } \\
\hline B & entire body \\
\hline $\mathrm{CK}$ & cheek \\
\hline $\mathrm{CN}$ & chin \\
\hline DIG & digits \\
\hline DOR & dorsal \\
\hline $\mathrm{F}$ & face \\
\hline FA & forearm \\
\hline FL & forelimb \\
\hline FO & foot \\
\hline $\mathrm{G}$ & gums \\
\hline GEN & genitals \\
\hline $\mathrm{HA}$ & hand \\
\hline $\mathrm{HE}$ & head \\
\hline HL & hindlimb \\
\hline $\mathrm{L}$ & lips \\
\hline LL & lower lip \\
\hline LTR & lower trunk \\
\hline MTR & middle trunk \\
\hline $\mathrm{NE}$ & neck \\
\hline OR & oral structures (teeth, gums, palate) \\
\hline PIN & pinna \\
\hline SH & shoulder \\
\hline SN & snout \\
\hline TA & tail \\
\hline $\mathrm{TE}$ & teeth \\
\hline TO & toes \\
\hline TON & tongue \\
\hline TR & trunk \\
\hline UL & upper lip \\
\hline UTR & upper trunk \\
\hline $\mathrm{W}$ & wrist \\
\hline
\end{tabular}

\section{Materials and Methods}

Three adult macaque monkeys (Macaca fascicularis, weight range, 2.6$7.0 \mathrm{~kg}$ ) were used to determine the organization of somatosensory fields in the lateral sulcus. At the beginning of each experiment, the animals were anesthetized with ketamine hydrochloride (average, $68 \mathrm{mg} / \mathrm{kg}$ ) and xylazine (average, $4 \mathrm{mg} / \mathrm{kg}$ ) intramuscularly. Regular use of ketamine in previous, noninvasive procedures in these monkeys may account for 
the unusually high initial dose of ketamine required. To maintain surgical levels of anesthesia (keep the animal areflexive), supplements of ketamine or sodium pentobarbitone (s.c. or i.v., $2.8 \mathrm{mg} / \mathrm{kg}$ ) were given throughout the experiment. A tracheal cannula was inserted to maintain a clear air passage, and fluids (physiological saline with $4 \%$ glucose) were administered through an intravenous cannula.

Once the animal was anesthetized, the midline scalp was incised, the temporal muscle retracted, and a large opening was made in the skull over the lateral sulcus and anterior parietal cortex. The dura was then retracted. An acrylic well was built around the opening and filled with silicone oil to protect the cortex, and to prevent desiccation. An enlarged photograph was made of the exposed cortex so that the location of electrode penetrations could be recorded relative to cortical vasculature for later reconstruction.

A tungsten-in-glass electrode $(1 \mathrm{M} \Omega$ at $1 \mathrm{kHz})$ was lowered into the cortex using a motor-driven stepping microdrive attached to a manipulator (Narishige). Electrodes had exposed tips of up to $30 \mu \mathrm{m}$ and gave excellent multiunit, and sometimes single unit recordings. In all cases, the upper bank, lower bank, and insula of the lateral sulcus, the caudal bank of the central sulcus, and the middle and lateral post central gyrus were explored electrophysiologically. For recordings in sulci, the electrode was advanced in 300-500 $\mu \mathrm{m}$ steps and neural recordings were made at each point. For gyral recordings, the electrode penetrations were placed approximately $500 \mu m$ aparl and recondings were made from neurons at a depth of 900-1100 $\mu \mathrm{m}$ from the cortical surface. We judged these distances between recording sites to be adequate to determine the organization of individual fields, because neurons in adjacent sites in cortex had partially overlapping receptive fields on the body. The electrode angle varied in the three cases. In two cases (MM7 and MM8), the electrode was advanced parallel to the walls of the lateral sulcus, and recordings were made tangential to the cortical layers (Fig. 2). In the third case (MM6), the electrode was advanced oblique to the cortical surface and surfaces within the lateral sulcus (Fig. 2). Over 1000 recording sites (688 of which were in the lateral sulcus) were used to determine the organization of fields in the lateral sulcus and adjacent portions of anterior parietal cortex.

Neural responses were amplified, filtered, and viewed on an oscilloscope and heard through a loudspeaker. Stimulation consisted of lightly tapping the skin with fine probes, stroking the skin with brushes, stroking the skin with a broad stimulus, gently displacing hairs, lightly pressing the skin, tapping body parts, and manipulating joints. In this way, receptive fields for neuronal clusters could be determined. Receptive fields were defined as the maximal area of the body that, when stimulated with a given stimulus, evoked a neural response. Sites in which neurons did not respond well to cutaneous stimulation were tested for responsiveness to auditory and visual stimulation. At some recording sites, neurons responded to both visual and somatosensory stimulation. However, our methods were not designed to determine if individual neurons within a cluster responded to bimodal stimulation. Although bilateral receptive fields have been reported previously for this region (e.g., Whitsel, 1969; Robinson and Burton, 1980), this was not systematically studied in the present investigation. Thus, the maps presented represent the contralateral body surface only. Electrolytic lesions (10 $\mu \mathrm{A}$ for $6 \mathrm{sec}$ ) were placed in strategic locations for later identification in histologically processed tissue.

When electrophysiological mapping was complete, the animal was transcardially perfused with $0.9 \%$ saline followed by $3 \%$ paraformaldehyde in phosphate buffer, and then $3 \%$ paraformaldehyde in $10 \%$ sugar phosphate buffer. The brains were soaked for approximately 15 hr in $30 \%$ sugar phosphate buffer. In the two animals in which the electrode was advanced parallel to the lateral sulcus (MM7 and MM8), the cortex was removed from the brainstem and thalamus, the sulci were gently pried apart, and the cortex was manually flattened between glass slides. Using a freezing microtome, these cortices were cut parallel to the cortical surface into 40 or $55 \mu \mathrm{m}$ sections. In the case in which the electrode was advanced perpendicular to the exposed cortical surface (MM6), the cortex remained attached to the brainstem and thalamus, and was sectioned coronally into $50 \mu \mathrm{m}$ sections. In the former cases, sections were stained for myelin (Gallyas, 1979), and in the latter case, alternate sections were stained for myelin, processed for cytochrome oxidase (Carroll and Wong-Riley, 1984) or stained for Nissl substance.

In all cases, the lesions made during the experiments were located in the processed tissue and electrophysiological results were related to architectonic boundaries. In cases sectioned tangentially, the series of sections was drawn using a stereomicroscope and camera lucida, and it

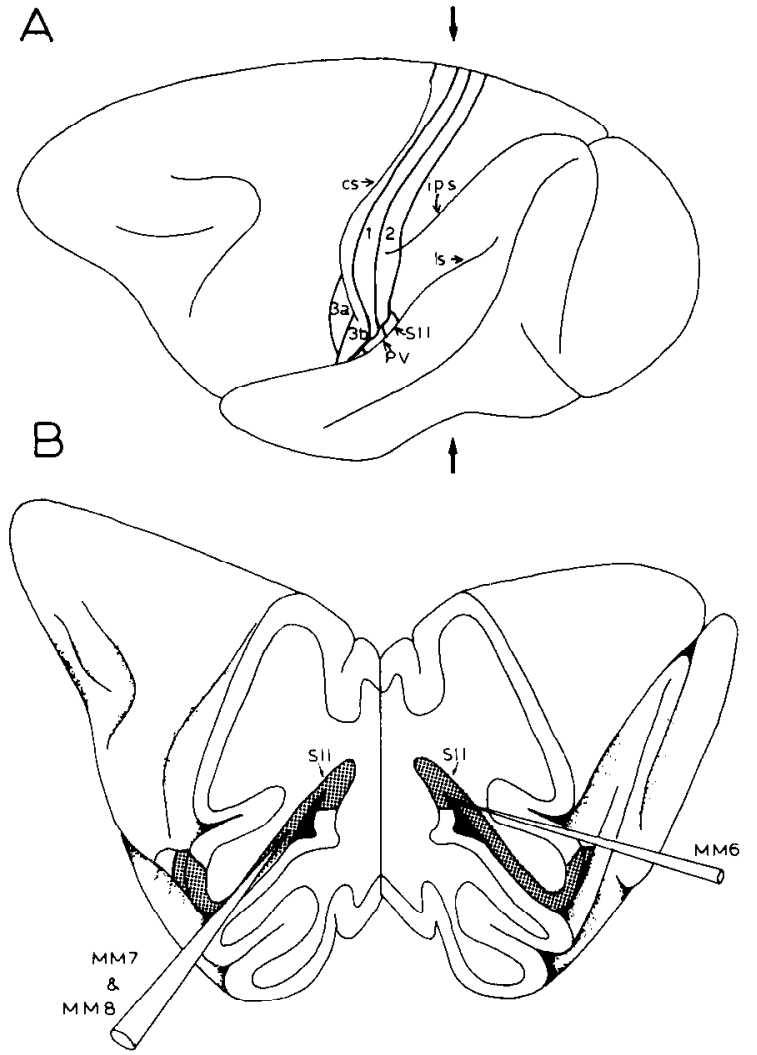

Figure 2. A dorsolateral view of the left cerebral hemisphere of the macaque monkey $(A)$, illustrating the position of anterior parietal and lateral parietal fields relative to the major sulci. Areas $3 \mathrm{~b}$ and $3 \mathrm{a}$ are in the posterior and anterior bank of the central sulcus, respectively, while areas 1 and 2 are located mostly on the dorsolateral aspect of the cortex between the central sulcus and the intraparietal sulcus. SII and PV were located immediately lateral to areas $3 \mathrm{~b}, 1$, and 2 . The large arrows indicate the position of the cut by which the brain was opened $(B)$. This figure also illustrates the angle of our electrode in the three recording experiments. In both $A$ and $B$, rostral is to the left and medial is to the top. See Table 1 for abbreviations.

was possible to identify all of the electrode tracks through the lateral sulcus, find the lesions, mark architectonic boundaries, and relate these to electrophysiological mapping data. In the case sectioned coronally, the series of sections was drawn and included electrode track damage, lesions, and architectonic boundaries. The sections were collated and geometrically rotated and "flattened" using the procedures described by Van Essen and Maunsell (1980). Although this method introduces large distortions when used to flatten the entire cortex, it produces fewer distortions when limited to a smaller region of cortex (i.e., lateral sulcus and insular region). By using this procedure in the case sectioned coronally, we were able to compare our cases with greater accuracy and to translate the topographic organization of areas in the lateral surface into two dimensional maps. The internal organization of fields derived using both methods were remarkably similar (compare Figs. 6A, 10A). In the following descriptions of the internal organization of cortical fields in the lateral sulcus, our directions refer to flattened maps of the cortex so that medial refers to superficial regions of the sulcus, and lateral to deep regions of the sulcus.

\section{Results}

In the present investigation, densely spaced recording sites (see Figs. $3 B, 6 B, 10 B$ ) in the lateral sulcus of macaque monkeys demonstrated that a large region of this cortex contained neurons responsive to somatic stimulation. An important observation was that in a number of distantly located rcgions of the lateral sulcus, the same body part was represented. Thus, regardless of how 




Figure 3. Cortical maps $(A)$ produced from recording sites $(B)$ in anterior parietal areas $3 \mathrm{~b}, 1$, and 2 , posterior parietal area 5 , and lateral sulcus areas SII and PV. Most of the mapping in SII and PV in this case was in the representations of the face, head, neck, and oral structures, located on the upper bank of the lateral sulcus, and in similar representations in lateral portions of the anterior parietal fields. The upper and lower banks 
distorted a sensory map might be, it was impossible to consider the lateral sulcus as containing a single cortical field.

The boundaries of cortical fields that we describe in the following results are determined using several criteria. The presence of a complete representation of the sensory epithelium was the strongest piece of evidence for segregating a region of cortex into a separate area. This was done by examining receptive field progressions, reversals and rerepresentations across regions of the lateral sulcus. Changes in the stimuli required to elicit a response, or in the response pattern, also helped delineate some of the boundaries of areas in the lateral sulcus. Finally, architectonic criteria combined with physiological distinctions were also used to determine the boundaries of cortical fields.

Using these criteria, we were able to delineate two complete topographic maps of the body surface. These two representations were essentially mirror reversals of each other. They share a common border formed by the representation of the digits of the hand and foot, and the representations of the lips and oral structures; the border was defined by a reversal in receptive field sequences. The organization, location, and position of the two fields with respect to anterior parietal cortex and each other, as well as the common boundary that they shared, is very similar to that described for fields SII and PV in the lateral sulcus of New World primates (e.g., Krubitzer and Kaas, 1990) and other mammals (e.g., Krubitzer and Calford, 1992). This suggests that the two fields are homologous (retained from a common ancestor) across mammals, and led us to term the two fields SII and PV in macaque monkeys. More limited mapping surrounding SII and PV indicated that at least two additional representations exist in surrounding cortex. We also explored the boundaries of anterior parietal fields (areas 3b, 1, and 2) with the fields in the lateral sulcus.

The relationship of anterior parietal and lateral somatosensory fields. Although previous maps of the lateral portions of areas 3b, 1, and 2 (Dreyer et al., 1975; Nelson et al., 1978; Pons et al., 1985) were more detailed than maps of this region generated in the present investigation, these studies did not map areas $3 b, 1$, and 2 in the same animal, nor were all of the recording sites in these studies as far lateral as in the present investigation (e.g., Nelson et al., 1978; Pons et al., 1985). Most important, these previous studies did not describe the relationships between lateral portions of anterior parietal fields and areas in the lateral sulcus.

In the present investigation, the lateral boundaries of areas $3 b$, 1 , and 2 were located just dorsal to the lip of the lateral sulcus, and at least two representations of the body surface within the lateral sulcus bordered these parietal fields laterally. As described in previous studies (e.g., Dreyer et al., 1975; Nelson et al., 1980; Pons et al., 1985), the face, lips, and oral representations in area 1 were caudal to those of area $3 \mathrm{~b}$, and the face, lip, and chin representations in area 2 were caudal to those of area 1 (Figs. $3 A, 6 A$ ). Figure 4 shows receptive field progressions through the face and lip representations in areas $3 \mathrm{~b}, 1$, and 2. Reversals in receptive field sequences were observed at the area 3 b/area 1 boundary and the area 1/area 2 boundary (Fig. 4 , receptive fields 1-8). Lateral to the face, lip, and oral representations of fields in anterior parietal cortex were similar representations in the lateral sulcus fields, as illustrated by the receptive field sequence A-E.

Rerepresentation and reversals in receptive fields for mediolateral recording site sequences originating in areas 1 and 2 demonstrated that anterior parietal fields shared a common mirrorreversal border with lateral parietal fields, and that these reversals coincided with architectonic distinctions (see below). As recording sites moved from medial to lateral in the lateral portion of area 1 , receptive fields moved from the representation of the chin onto the representations of the lips, tongue, and teeth, reversed at the area 1/area PV boundary, and moved from the tongue representation onto the lip and chin representations (Fig. 5 , receptive fields $A-G$ ). Likewise, as recording sites moved from medial to lateral in area 2 , corresponding receptive fields for neurons in those sites moved from the representation of the lips, onto the representations of the chin, face, and neck laterally. As the area 2/SII boundary was crossed, receptive fields reversed and moved from chin, neck, and face, back to the lips (Fig. 5, receptive fields $1-7$ ).

The organization of SII and PV. The arca of cortcx responsive to somatic stimulation in the lateral sulcus was very large $(\sim 150$ $\mathbf{m m}^{2}$ ), and appeared to contain at least two representations of any given body part. By considering the number of complete representations of the body surface, and receptive field sequences and reversals, we divided this responsive region into two fields. The relationship of the two fields to each other is best appreciated when receptive field progressions are drawn through both fields, and reversals in the sequence, and duplications in representations of body parts are revealed (Fig. 4, receptive fields A-E; Figs. 7-9).

The field designated SII was adjacent to the lateral boundary of areas 1 and 2, and the field termed PV was immediately rostral to SII, and adjacent to the lateral boundary of areas $3 b$ and 1. The mediolateral sequence of organization in SII and PV were similar. In both fields, the lips, oral structures, and face were represented most superficially on the upper bank of the lateral sulcus (UBLS) or medially in cortex that had been flattened (Figs. 3,6). The representations of the forelimb were just lateral to the representations of the face or deeper in the sulcus in the intact brain. The representations of the hindlimb were located at the deepest portion of UBLS and, in SII, sometimes spread onto the fundus of the lateral sulcus. The trunk representation of SII was located caudal to the representation of the limbs, and the trunk representation in PV was just rostral to the representation of the limbs (Fig. 6A). Thus, the somatotopic organization of SII could be described as a noninverted homunculus with respect to the brain, while that of PV could be described as an inverted homunculus.

Within the face representation of SII, the lips were represented most medially and were adjacent to the lip and face representations of areas 2 and 1 . The chin, face, and snout representations surrounded the representation of the lips (Figs. $3 \Lambda, 6 \Lambda$ ). The organization of the face representation was determined in

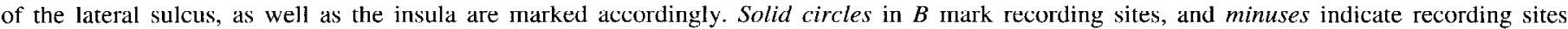

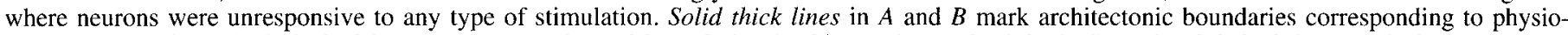

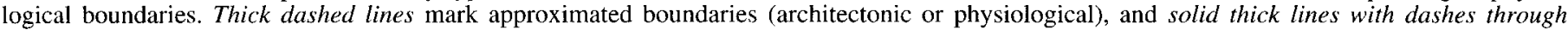

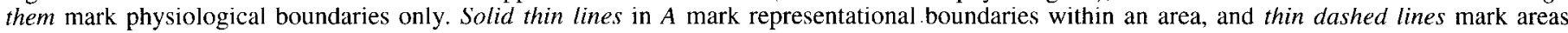
where the density of mapping was low. See Table 1 for abbreviations. 

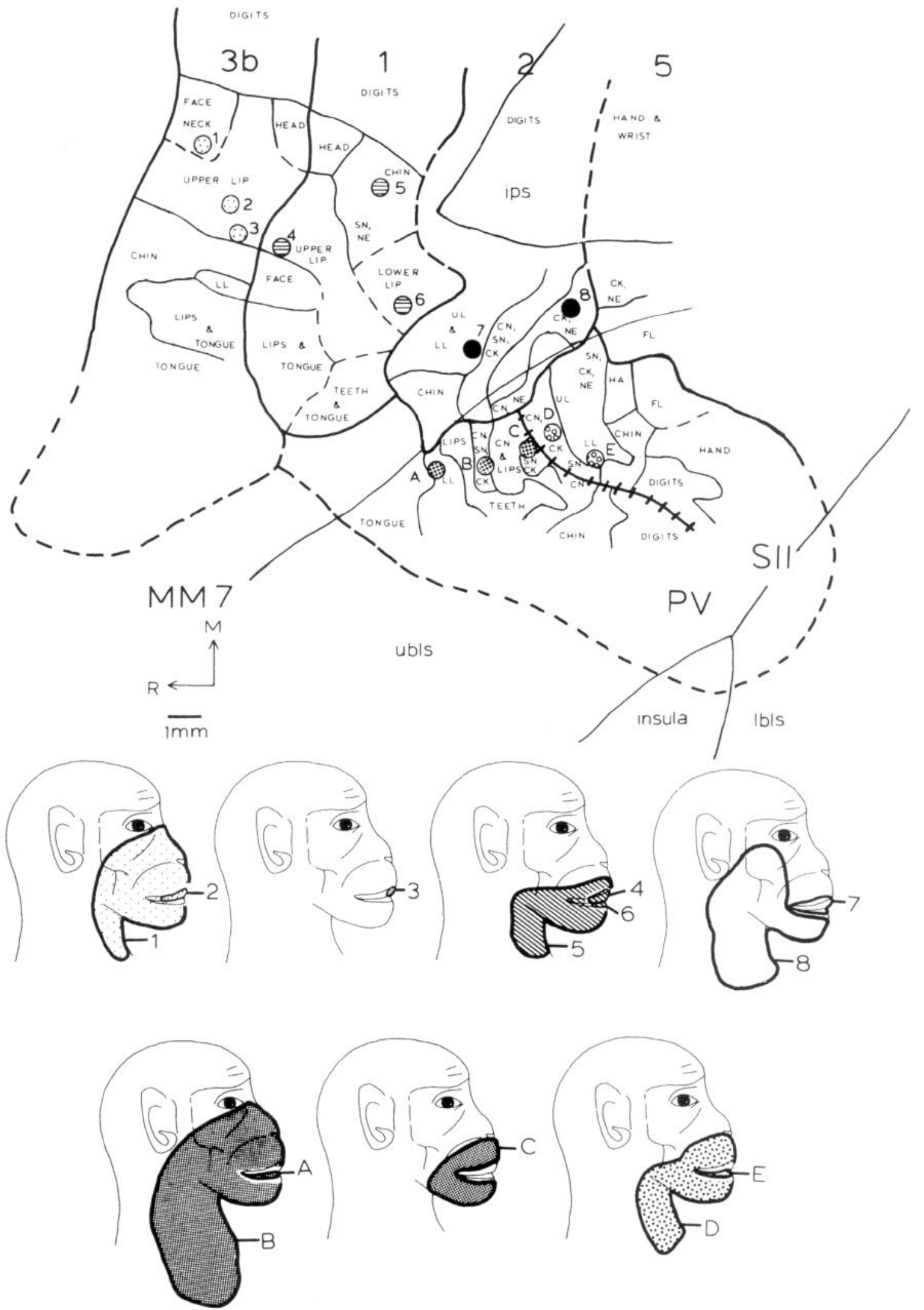

Figure 4. The top panel illustrates maps of anterior parietal and lateral sulcus areas in MM7. Recording site progressions through anterior parietal fields are numbered, and recording site progressions through lateral sulcus fields are lettered. The type of stipple used for recording sites in a given area in cortex matches that for the corresponding receptive field on the body part drawings. A reversal in receptive field progression $(A-E)$ is observed at the PV/SII boundary. Conventions are as in Figure 3 and Table 1. 


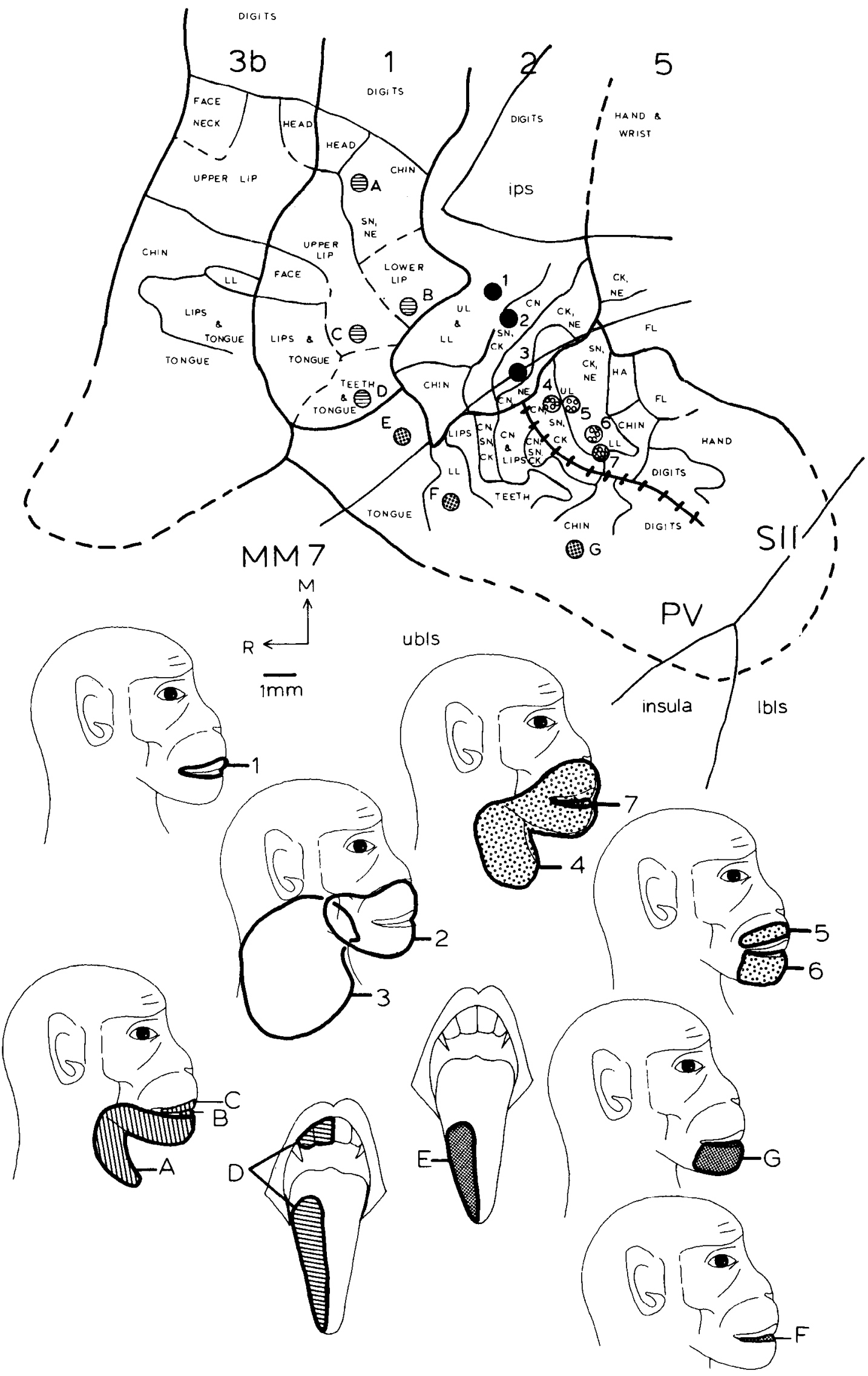

Figure 5. The top panel illustrates a simplified map of anterior parietal and lateral sulcus areas in MM7 showing recording site progressions from area 1 into PV $(A-G)$, and from area 2 into SII $(1-7)$. The type of stipple used for recording sites in a given area in the cortex matches that for the corresponding receptive field on the body part drawings. Conventions are as in Figure 3 and Table 1. 




Figure 6. Cortical maps $(A)$ produced from electrophysiological recording sites $(B)$ for areas in both anterior parietal cortex and the lateral sulcus in MM8. Most of the recording sites were in SII and PV. In both SII and PV, the same mediolateral sequence of organization is observed with the face represented most medially, followed by the forelimb and hindlimb laterally. However, the rostrocaudal organization of both fields is reversed so that they form mirror symmetric representations. Conventions are as in Figure 3 and Table 1.

greatest detail in one animal (Figs. 3-5). Within PV, the tongue, lips, and teeth were represented rostromedially, and the chin, snout, and cheek were represented caudomedially and laterally (Fig. 3A). In one case, the representations of the head, neck, face, and pinna were found to lie caudolateral to the oral representations (Fig. 10A). Recording site sequences through the head and face representations of SII show that with a progression from caudal to rostral in cortex, receptive fields moved from the head, neck, and upper trunk, to the rostral snout and chin, and into the mouth (Fig. 7, receptive fields 1-3; Fig. 4, receptive fields D and E). As recording sites crossed the SII/PV boundary, receptive fields for neurons in those sites reversed and moved from the chin, neck and cheek onto the chin, neck, upper trunk, and shoulder (Fig. 7 , receptive fields 4 and 5), or onto the lips (Fig. 4, receptive fields A-C).
The trunk representation was found most caudal and lateral in SII. In the middle region of SII, the forelimb representation was located just rostral to this, and the distal portion of the forelimb, hand, and digits were represented most rostrally in the field. Within the hand representation of SII, the digits were represented most rostrally in the field, and were surrounded by representations of the palmar surface (Figs. 6A, $10 A$ ). Radial portions of the hand were represented more medially, and ulnar portions of the hand laterally. Hairy portions of the hand were represented lateral and/or caudal to the digit representation, and receptive fields for neurons in this region also included more proximal portions of the dorsal and ventral forelimb. Within PV, the upper trunk was represented rostrally, more proximal portions of the limbs were represented medially and caudally, and the distal forelimb and digits were 


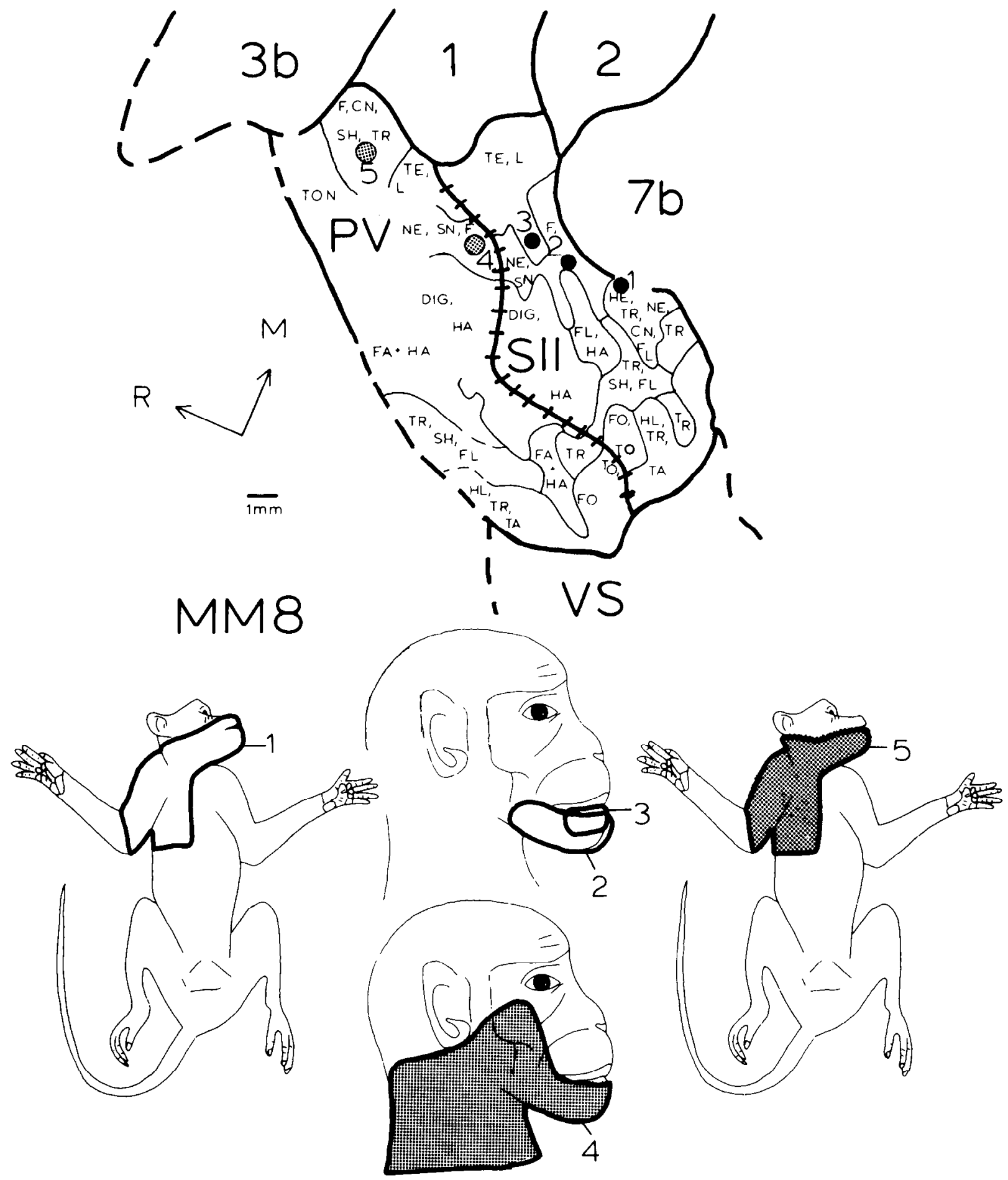

Figure 7. A simplified version of the map produced for MM8 with recording site progressions through SII and PV (top), and corresponding receptive fields for neurons in those recording sites (bottom). The stippled and solid circles in the top panel correspond to stippled and open receptive fields, respectively, in the bottom panel. Recording site progressions through the upper trunk and face regions in both SII and PV show a reversal at the SII/PV boundary. Note that very similar receptive fields ( 1 and 5 ) are observed for neurons at very distant locations in cortex, rostrally in PV and caudally in SII. Conventions are as in Figure 3 and Table 1.

represented most caudal, adjacent to the representation of the digits in SII (Figs. 6A, 10A). As in SII, the representation of the digits of the hand was surrounded by the representations of the hairy hand and forelimb. Also, the radial hand was represented medial to the ulnar hand. As recording sites in cortex moved from caudal to rostral within the forelimb rep- resentation of SII, corresponding receptive fields for neurons in those sites moved from the midline of the upper trunk, onto proximal portions of the limb, and then onto distal portions of the hand and digits (Fig. 8, receptive fields 1-4). As recording sites crossed the SII/PV boundary and moved from caudal to rostral in the forelimb representation in PV, corre- 


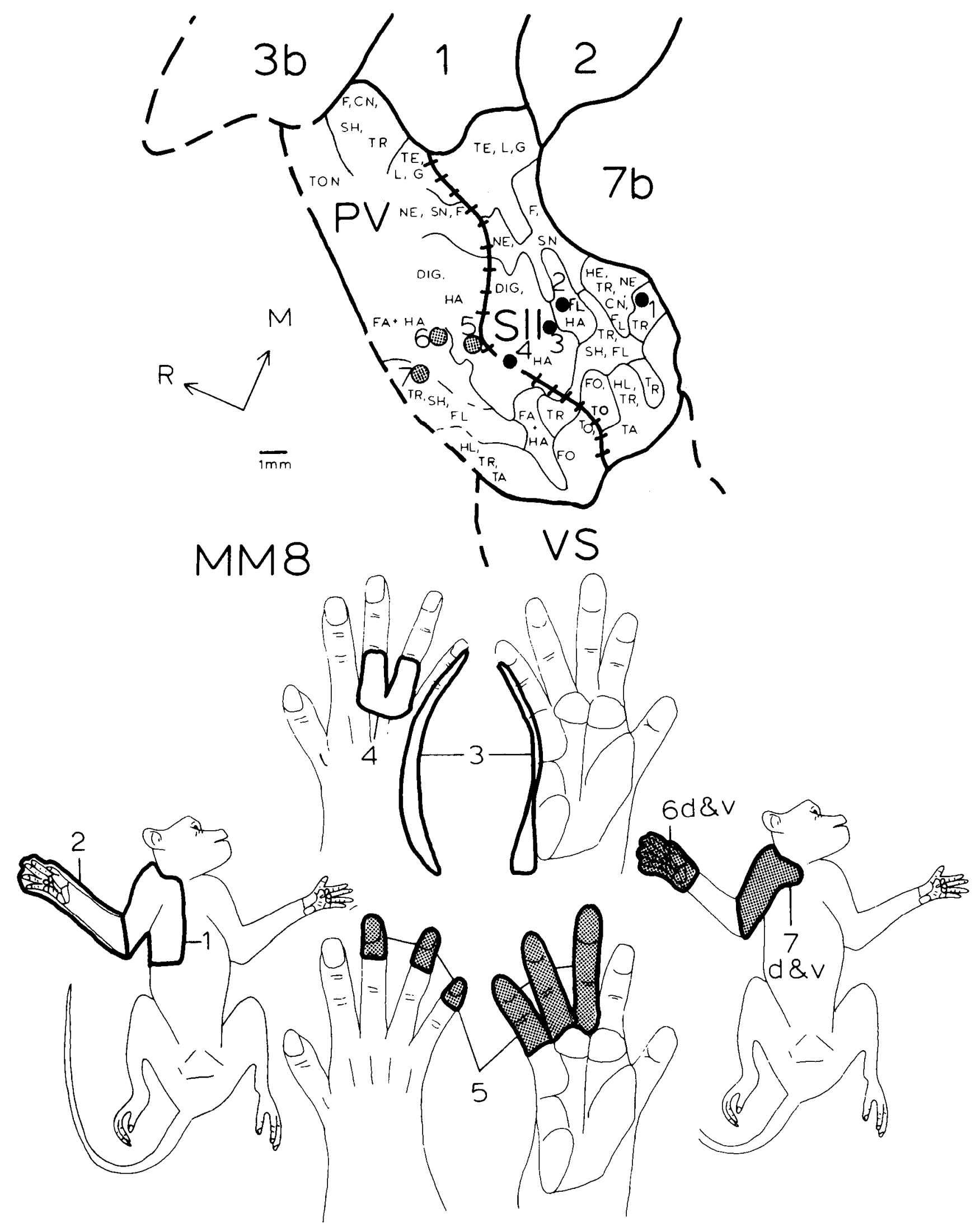

Figure 8. Receptive fields (bottom) for a progression of recording sites through the forelimb and upper trunk representations in SII and PV in MM8 (top). As recording sites move from caudal to rostral in SII, corresponding receptive fields for neurons in those sites move from upper dorsal and ventral trunk and shoulder, onto the forelimb, hand and digits (receptive fields 1-4). As recording sites cross the SII/PV boundary, receptive fields for neurons reverse and move from the digits, onto the hand, and then onto the dorsal and ventral forelimb, shoulder, and upper trunk (receptive fields 5-7). $d$, Dorsal; $v$, ventral. Other conventions are as in Figure 3 and Table 1. 
sponding receptive fields for neurons in those sites moved from distal to proximal (Fig. 8, receptive fields 5-7).

The representation of the hindlimb was bounded caudally by the representation of the lower trunk, and laterally, by that of the trunk and tail (Figs. 6A, 10A). The representations of the proximal hindlimb and tail were located more laterally in the flattened cortex, or in the fundus of the lateral sulcus. The representation of the distal hindlimb was located rostrally, or rostromedially within the overall hindlimb representation. Within the hindlimb representation of PV, the lower trunk, or lower trunk, hindlimb and tail, were represented rostrally; the proximal hindlimb was represented more caudally, and the foot and toes were represented most caudally, adjacent to the representation of these parts in SII (Figs. 6A, 10A). A representation of the genitals was found most laterally in PV (Fig. 10A). In both cases in which the hindlimb/trunk representation of PV was mapped, it was found to spread onto the insula of the lateral sulcus (Figs. $6 A, 10 A$ ). As recording sites progressed from caudal to rostral in the hindlimb representation in SII, corresponding receptive fields moved from the dorsal midline of the lower trunk and tail, to proximal hindlimb, to the distal hindlimb, foot, and toes most rostrally (Fig. 9, receptive fields $1-3$ ). As recording sites crossed the SII/PV boundary and moved from caudal to rostral in PV, receptive fields for neurons in these sites moved from the foot and toes, to hindlimb, to lower trunk, and tail (Fig. 9, receptive fields 4-6).

Neurons in both SII and PV responded well to cutaneous stimulation. Although both rapidly adapting and slowly adapting response patterns were noted, this response property was not systematically examined. Most often, neurons in both fields could be activated by lightly tapping or brushing the skin, although some neurons required more intense stimulation such as light taps, pressure, or joint manipulation. Receptive fields for neurons in both fields varied in size, depending on the body part being stimulated. Receptive fields for neurons that represented the trunk were generally large and often incorporated adjacent portions of the proximal limb (e.g., Fig. 9, receptive fields 1 and 5 ), while receptive ficlds for ncurons on the lips and digits could be quite small (e.g., Fig. 5, receptive fields 7 and F; Fig. 8, receptive fields $3-5$ ), and often comprised only a single digit tip (not shown), or a portion of the upper or lower lip.

Cortex surrounding SII and PV. With the exception of cortex rostral to PV, cortex surrounding SII and PV contained neurons responsive to somatosensory stimulation. In our preparation, cortex rostral to PV was unresponsive to somatic stimulation. In cortex caudal and/or medial to SII, within the area $7 \mathrm{~b}$ region described in previous investigations (e.g., Robinson and Burton, 1980; Andersen et al., 1990; Neal et al., 1990), neural responses to cutaneous stimulation were of longer latency than neurons in SII and PV. Receptive fields were often very large, and a broad moving stimulus across the entire receptive field was required to evoke a neural response. In one case, MM6 (Fig. 10), neurons in medial portions of area $7 \mathrm{~b}$, caudal to area 2 , were unresponsive to sensory stimulation under the present recording conditions. In addition to the differences in neural responses and receptive field size, the presence of an additional forelimb and hand representation, approximately $3 \mathrm{~mm}$ from a similar repre- sentation in SII, and separated from the hand representation in SII by the head, neck and trunk representations, indicated that this region was part of a separate field (Fig. 6A).

There was also evidence for another field lateral to SII and PV, which was located on the insula, fundus, and lower bank of the lateral sulcus. This field was termed VS, because its location and organization were similar to those of VS described in owl monkeys (Cusick et al., 1989) and flying foxes (Krubitzer and Calford, 1992). However, more extensive mapping, architectonic analysis and data on this region's connections are needed to establish homology. Neurons in VS generally had larger receptive fields than those of neurons in SII and PV, and VS contained representations of body parts (e.g., foot, Fig. $6 B$; upper trunk and hindlimb, Fig. 10B) located several millimeters away from similar representations in SII and PV.

In one case (MM8, Fig. 6), the cortex caudal and lateral to SII and lateral to area $7 \mathrm{~b}$, in the location of the retroinsular area, $\mathrm{Ri}$, of other investigations (see Burton and Robinson, 1981), was found to contain neurons responsive to visual or visual and somatosensory stimulation. These neurons were easily distinguished from those of surrounding fields because they responded less vigorously to somatic stimulation, had large, ill-defined receptive fields, and were located outside the moderately myelinated SII and PV region (see below).

Cortical architecture. The architecture of SII, PV, and surrounding cortex was examined in tangentially sectioned cortex stained for myelin (Fig. 11). To determine cortical field boundaries, an entire series of sections through the cortex was reconstructed and superimposed so that irregularities due to flattening could be appreciated, and the entire extent of fields determined. In the highly fissured macaque monkey cortex, the extreme curvature of major sulci results in differential staining across areas that straddle the lip or fundus of a sulcus (e.g., Fig. $11 B$ ).

In this preparation, both SII and PV stained moderately for myelin and were distinguished from lightly myelinated cortex rostrally, laterally, and caudally (Fig. I I). Thus, myeloarchitecture was most useful for distinguishing SII and PV from surrounding cortex, although not from each other. Anterior parietal fields $3 \mathrm{~b}, 1$, and 2 could also be distinguished from each other and from areas SII and PV on the basis of myeloarchitecture. As reported previously (e.g., Nelson et al., 1978), area 3b stained densely for myelin, especially in middle cortical layers, while area 1 was more moderately myelinated. Although the boundary between the moderately staining area 1 and lightly staining area 2 could be delinited in our preparation, the caudal boundary of area 2 was not always distinct.

In coronally sectioned tissue stained for Nissl substance (Fig. 12), SII and PV contained a densely packed layer IV compared to surrounding cortex, as has been described for the SII region in previous investigations (e.g., Friedman et al., 1986). SII contained a somewhat more densely stained layer VI than did PV (Fig. 12A,B). However, a parasagittal plane of section would be required to relate this difference to physiological maps. Nissl stains were also useful in distinguishing lateral somatosensory areas (SII and PV) from anterior parietal fields, especially SII from area 2, which contained lightly stained and loosely packed infragranular layers; these distinctions were especially clear in

Figure 9. Recording site progressions through the representations of the lower trunk, hindlimb, and foot in both SII and PV (top), and corresponding receptive fields for neurons in those sites in MM8 (bottom). Similar receptive fields (e.g., 1 and 6 ) are found in very distantly located portions of cortex in areas SII and PV, respectively. Conventions are as in Figure 3 and Table 1. 

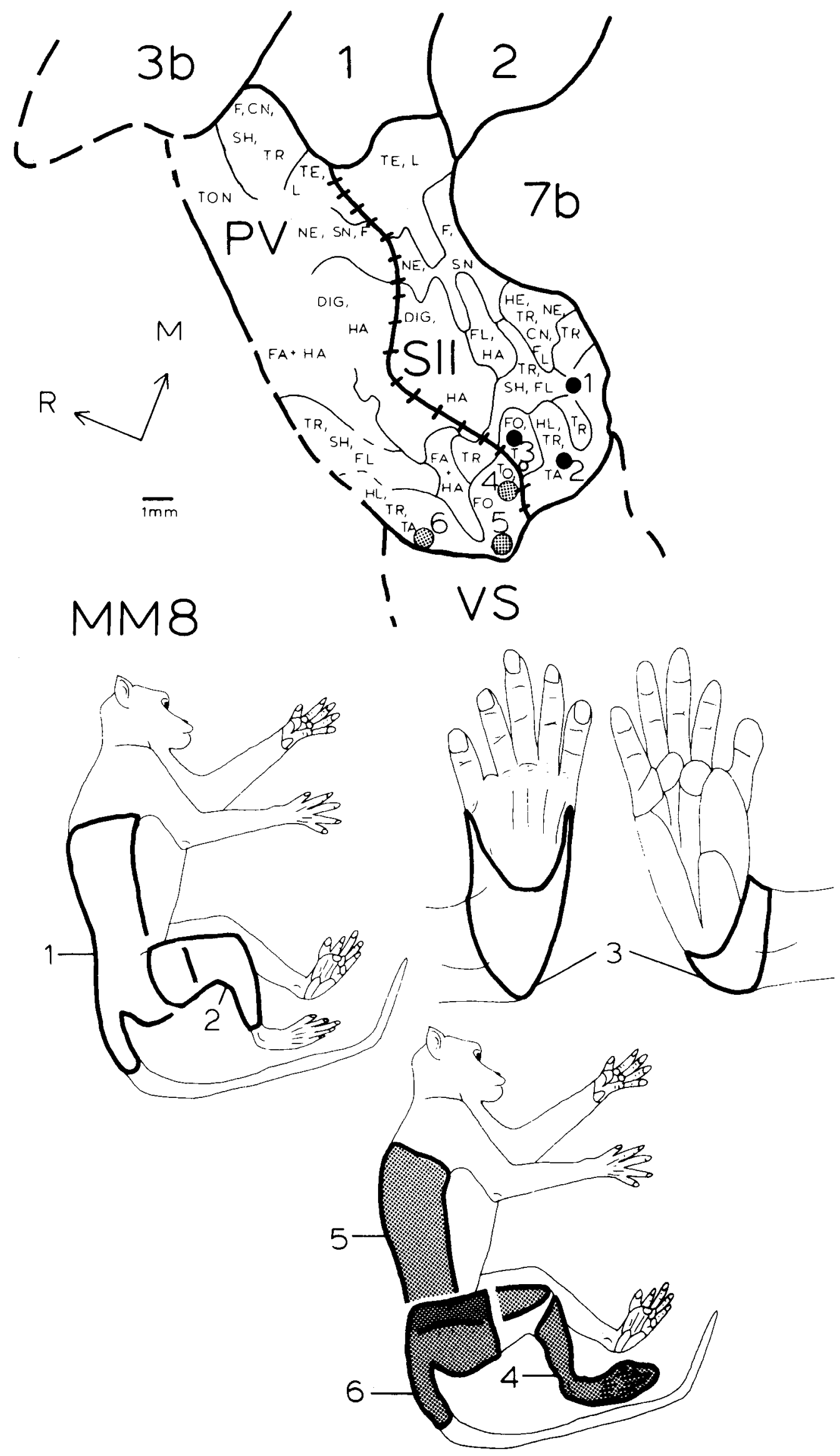


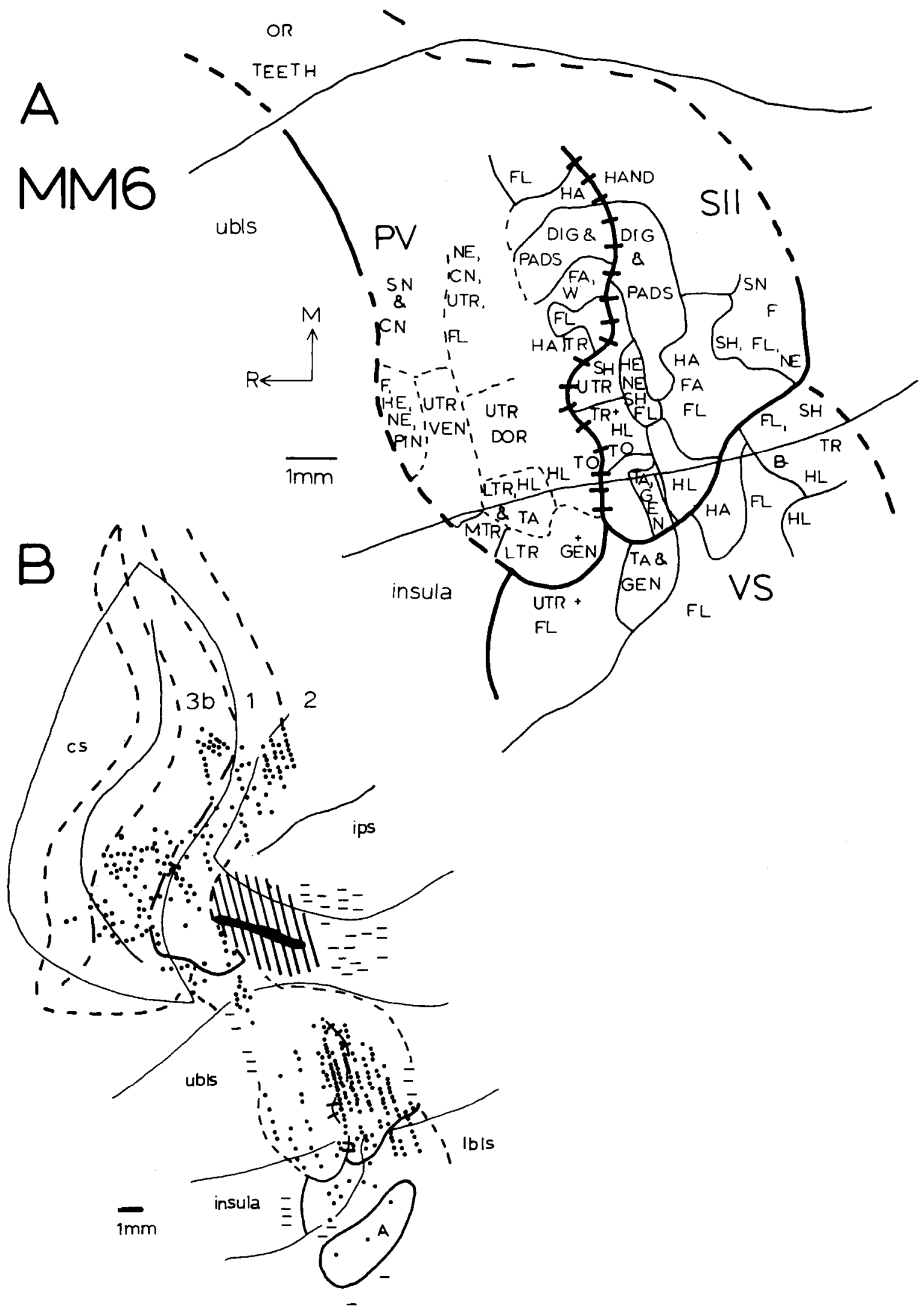

Figure 10. Cortical maps $(A)$ produced from electrophysiological recording sites $(B)$ in anterior parietal and lateral sulcus areas in MM6. Most of the mapping in the latcral sulcus areas was in the lateral portions of SII, with more limited mapping in PV and VS. As in the previous cases, the mediolateral organization of SII and PV was similar, with the face represented most medially followed by the forelimb and finally the hindlimb most laterally. The two fields formed a common border at the representations of the digits of the hand and foot. In $B$, the lined region indicates an area of cortex that was damaged in which neurons responded poorly or were unresponsive to stimulation. Conventions are as in Figure 3 and Table 1. 

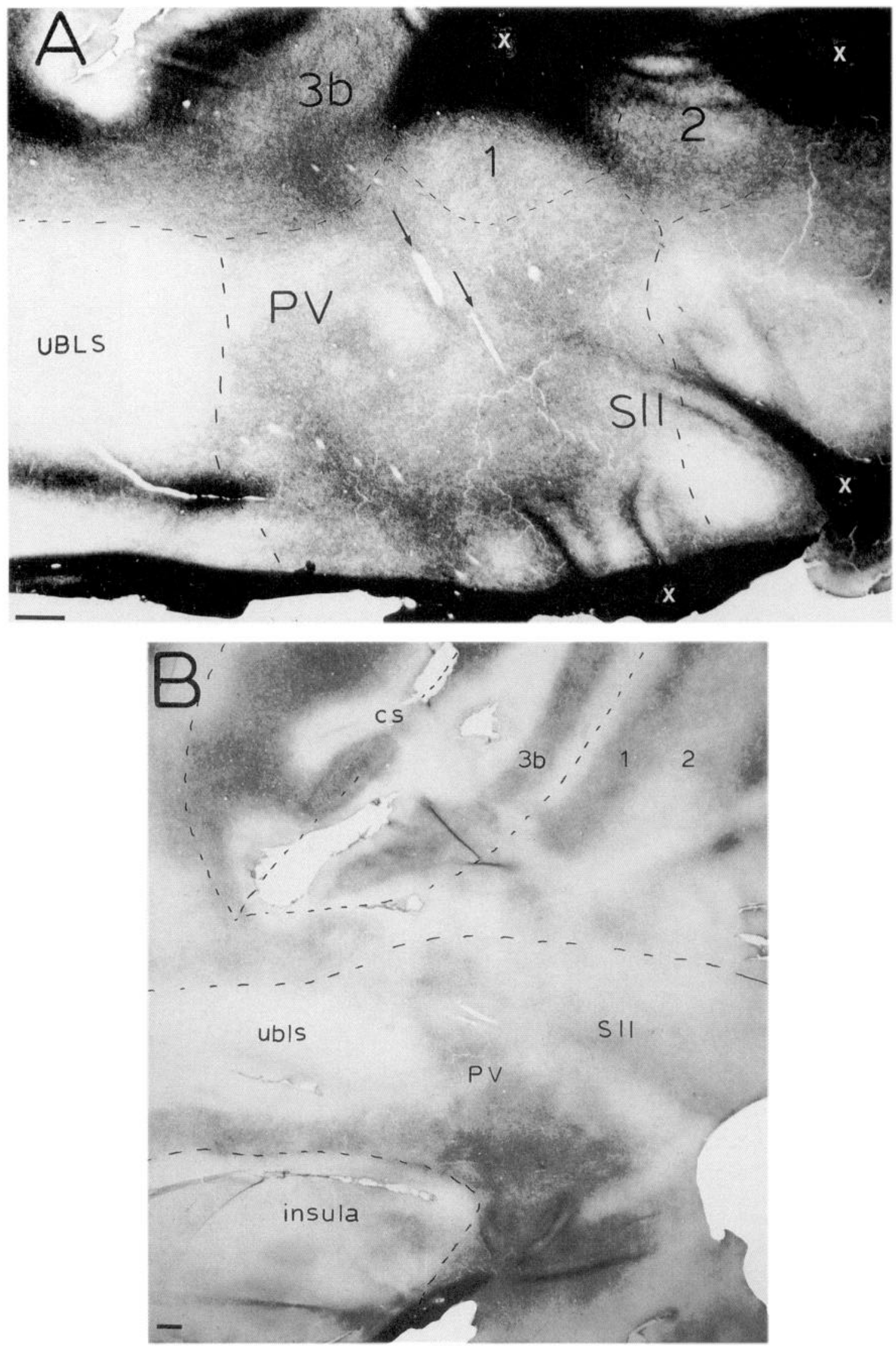

Figure 11. Light-field photomicrographs of cortex that has been flattened, cut parallel to the cortical surface, and stained for myelin in MM8 (A), and a nonexperimental monkey $(B)$. Both SII and PV stain darkly for myelin, while cortex rostral, and caudal to these fields stained more lightly. In $A$, cortex lateral and medial to SII and PV stained very densely since the section here is through the white matter (white $X^{\prime} s$ ). More lateral 
coronally sectioned tissue (Fig. 12A, star). Cytochrome oxidase staining did not prove useful for distinguishing subdivisions of the lateral sulcus.

\section{Discussion}

These results provide physiological evidence for two complete representations of the body surface, SII and PV, in the region of cortex traditionally defined as SII. However, further studies of architecture, connections, and single unit response properties are required to support the differences between these fields. In this study, we have termed the caudal field SII and the rostral field PV for historical reasons, and to remain consistent with previous studies on other species. The observation that descriptions of SII varied across studies was first made by Herron (1978) who described a noninverted representation of the body surface in raccoons as SII. Until this time, SII was depicted as an inverted representation. However, early investigations of SII using evoked potential recordings (Woolsey and Fairman, 1946; also see Woolsey, 1958) did not provide a detailed description of the organization of SII, only a summary homunculus (see Fig. 22 of Woolsey, 1958), which assumed a very large region of the lateral sulcus. Thus, while Woolsey coined the term SII, his study indicated only the existence of an additional representation, not its detailed organization. Complete and detailed de scriptions of SII were subsequently provided by fine-grained, microelectrode mapping studies in a number of mammals such as raccoons, cats, mice, squirrels, agoutis, tree shrews, and rabbits, and in all of these investigations, SII was described as a noninverted representation, as in the present study (see Johnson, 1990 , for review). Furthermore, in primates in which SII was mapped in detail (galagos, Burton and Carlson, 1986; owl monkeys, Cusick et al., 1989; marmosets, Krubitzer and Kaas, 1990) it had a very similar organization to that of the caudal area, termed SII in the present study. PV was first described in squirrels as an inverted representation (Krubitzer et al., 1986), and in other species in which both SII and PV have been delineated in the same animal (e.g., Fabri and Burton, 1991, rats; Krubitzer and Calford, 1992, flying foxes), the internal organization and relationship between the two fields are similar to those found in the present investigation in macaque monkeys (Fig. 13). We believe that SII and PV in macaque monkeys are homologous to SII and PV described in other mammals, and are components of a basic plan of mammalian somatosensory cortical organization, that may exist in humans as well. Support for this latter proposition comes from a recent investigation in humans using positron emission tomography, in which two representations of the hand and foot were identified in the lateral sulcus region (Burton et al., 1993). However, the centers of the foci of activity were separated by a relatively large amount of cortex.

Previous reports on SII in macaque monkeys vary in their descriptions of the size and internal organization of the field (Fig. 1). However, when previous and present maps of SII are examined, all observations are consistent with our finding of two adjacent, mirror symmetric representations. In two of these previous reports, it is likely that the proposed SII contained portions of both SII and PV of the present investigation (Whitsel et al., 1969; Robinson and Burton, 1980). Indeed, in their investigation of SII, Robinson and Burton (1980) proposed that this region may actually contain more than one field. Furthermore, connections from different body part representations of anterior parietal fields are to two separate locations in the lateral sulcus, in the approximate location of SII and PV of the present study (see Figs. 9-12 of Friedman et al., 1980; Alloway et al., 1990). Finally, the area termed SII in one study (Pons et al., 1988) appears to correspond to our rostral representation, PV (compare Figs. $1 B, 6 A$ ).

Limited electrophysiological recording data indicate the existence of additional fields in cortex adjacent to SII and PV. Previous investigations of the insular region in macaque monkeys have reported that it contains neurons responsive to cutaneous stimulation (e.g., Robinson and Burton, 1980; Burton and Robinson, 1981; Schneider et al., 1993). It would appear that Schneider et al. (1993) were recording from neurons in the region we term VS. Furthermore, Burton and Robinson (1981) describe a large region of cortex surrounding SII as containing neurons responsive to somatic stimulation. Some of the surrounding rcgions in thcir study appear to correspond to what we term VS, and cortex just caudal to SII in the present investigation. However, further work is needed to clarify this. A number of investigations have used architectonic criteria to subdivide the lateral sulcus and insular region, and have devised a scheme which includes areas designated Ig, Id, Pa, Pi, and Ri (e.g., Burton and Robinson, 1981; Friedman et al., 1986). The maps generated in the present investigation do not appear to correlate with these previously proposed subdivisions.

Another issuc that arises from the present results relates to the relationship between the anterior parietal and lateral parietal somatosensory areas. Generally, areas 1 and 2 are depicted as curving under area $3 b$ ventrally, making SII adjacent to the far ventral boundary of area 2 only (see Fig. 4.2 of Burton and Robinson, 1981). However, our results indicate that areas 1 and 2 do not wrap around area $3 b$ ventrally, but border the lateral fields at the lip of the lateral sulcus. In comparison with the description in other mammals, SII is somewhat caudal in the macaque. The large expansion of both anterior parietal cortex and the cortex of the lateral sulcus in Old World primates may have produced a displacement of this field.

In addition to issues of homology and spatial relationships of somatosensory fields, our results have important implications for studies describing sensory processing networks. It has been proposed that there exists a separate simian scheme of cortical and subcortical processing in which a stricter hierarchy is observed, making SII dependent upon SI for activation (e.g., Garraghty et al., 1991). In an effort to determine the hicrarchical relationship of cortical areas in serial processing streams, studies which de-

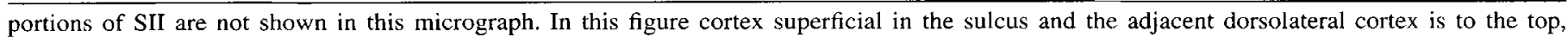

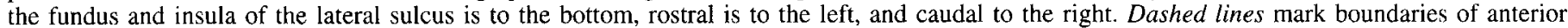

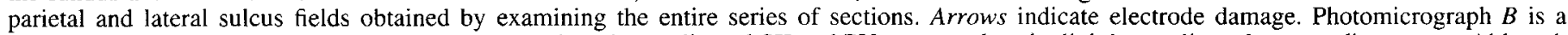

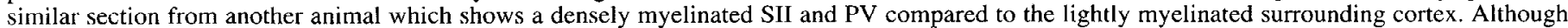

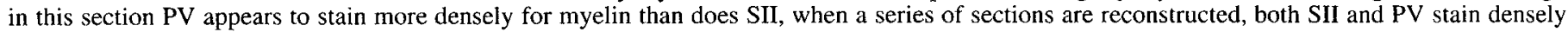

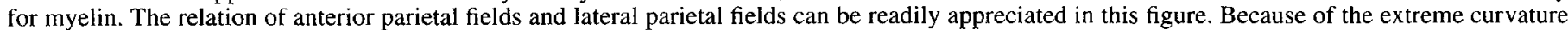

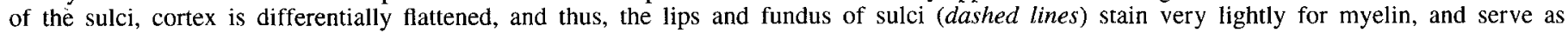
consistent cortical landmarks. Scale bars, $1 \mathrm{~mm}$. Abbreviations are in Table 1. 

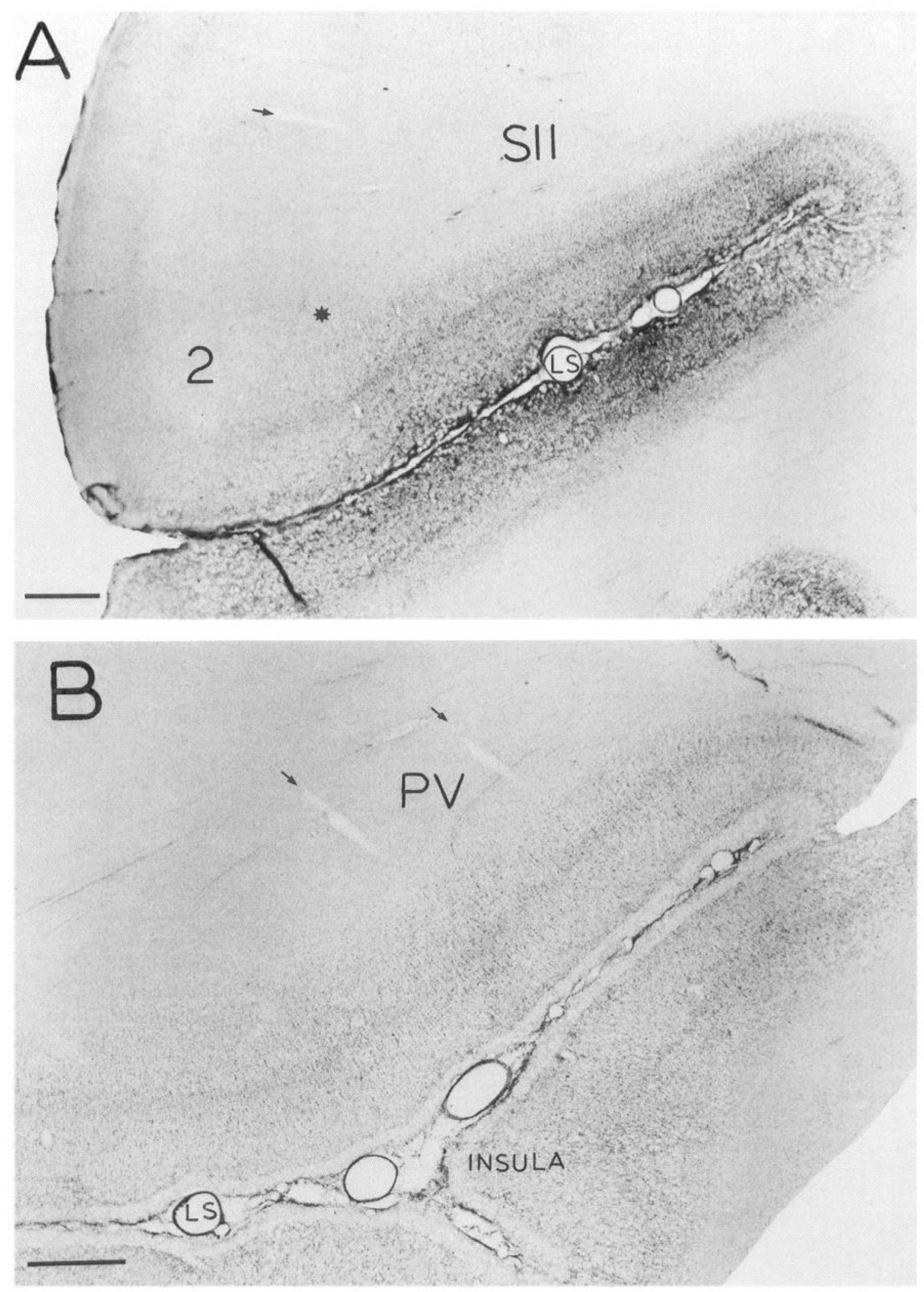

Figure 12. Coronally sectioned tissue stained for Nissl substance in SII $(A)$ and PV $(B)$ from MM6. The arrows indicate electrode tracks through the trunk representation in PV and the hand representation in SII. When sections in the series were collated, all the electrode tracks could be readily identified. In this preparation, both SII and PV have a densely packed, darkly staining granule cell layer (IV), and SII contains a somewhat more densely staining layer VI. The boundary between area 2 and SII is marked (star) by a decrease in cell packing and staining in infragranular layers. Scale bars equal $1 \mathrm{~mm}$. The dorsolateral surface of cortex is to the left, and the fundus and insular region of the lateral sulcus are to the right. Abbreviations are in Table 1. 



Head, Neck \& Face
Trunk

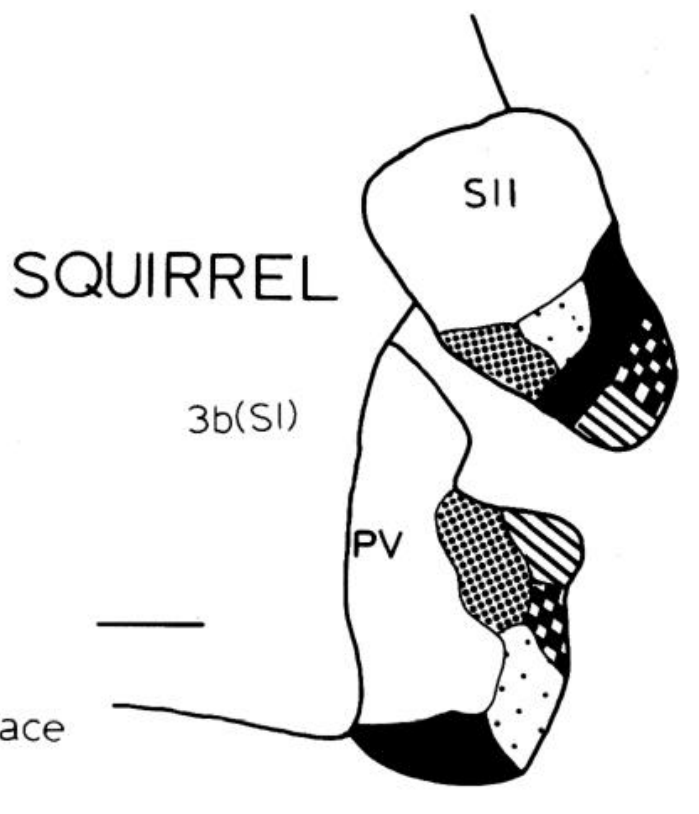

Distal Forelimb

(:) Proximal Forelimb

(1) Distal Hindlimb

- Proximal Hindlimb

Figure 13. Summary maps of the organization of SII and PV in Old World macaque monkeys, New World marmoset monkeys, flying foxes, and squirrels. Although there is some variation in the details of the organization of the two fields, in all of these species SII and PV form mirror symmetric representations of each other. In all species but the squirrel, the two fields share a common boundary at the representation of the digits, toes and portions of the face; the trunk representation is located at the outer boundaries of these fields. In squirrels SII and PV are separated by a thin strip of cortex in which neurons are unresponsive to sensory stimulation. The summary of the marmoset is modified from Krubitzer and Kaas (1990), that of the flying fox is modified from Krubitzer and Calford (1992), and that of the squirrel is modified from Krubitzer et al. (1986). The macaque monkey summary is a compilation of all of our cases, based mainly on MM8. Scale bars, 1 mm. Conventions are as in Figure 3 and Table 1 . 
activate fields at the beginning of supposed cortical hierarchies and then examine the effects on fields at higher hierarchical levels have been carried out in a variety of mammals and sensory systems. In mammals such as cats, rabbits, tree shrews, and galagos, lesions or cooling of SI have little effect on SII neurons (Manzoni et al., 1979; Burton and Robinson, 1987; Burton et al., 1988; Garraghty et al., 1991; Turman et al., 1992). In contrast, in primates such as macaque monkeys (Pons et al., 1988; Burton et al., 1990) and marmosets (Garraghty et al., 1990), cooling or lesioning SI "deactivates" SII. However, a recent investigation in marmosets, in which neural responses in SI were effectively blocked by cooling, demonstrated no loss of responses in the majority of neurons in SII (Zhang et al., 1993).

The differences between studies could result from a number of factors. First, SI has not been consistently defined across studies. A number of investigators persist in calling areas 3a, 3b, 1 , and 2 in primates "SI" (e.g., Carlson and Nystrom, 1994), or treating these fields as a single field (Lund et al., 1993), despite the overwhelming evidence that each constitutes a separate field. Only area $3 \mathrm{~b}$ should be considered homologous to SI in other mammals (Kaas, 1983), and the inconsistent use of the term is especially misleading when comparing primates with non-primate mammals. In some experiments only area $3 b$ was deactivated, while in other investigations areas $3 \mathrm{a}, 3 \mathrm{~b}, 1$, and 2 , or various combinations of these four fields, were deactivated. Yet most studies report that "SI" was deactivated. Second, the target field may have been misidentified. In some studies it is likely that what we term SII was mapped (Garraghty et al., 1990), but in others it seems probable that the field that we term PV was mapped (e.g., Pons et al., 1988), since the size and internal organization are consistent with the description of PV in the present study. Only one investigation distinguished SII from PV after anterior parietal ablations (Garraghty et al., 1990). While there may indeed be separate simian and non-simian plans of somatosensory processing, related to differences in thalamic and cortical connections across species, the details of the differences cannot be resolved until issues of homology are addressed. A broad range of mammals needs to be examined in detail to ascertain which features of cortical organization are retained in all lineages, and which features are specialized. It would then be possible to determine how retained features (e.g., SI and SII), and their function, have been modified with the addition of new cortical fields in groups such as primates.

In this study, we found that the basic plan of organization of lateral somatosensory areas in macaque monkeys is common to that found in many other eutherian mammals. Despite a massive enlargement of this area to more than $150 \mathrm{~mm}^{2}$, it is dominated by two complete representations of the cutaneous body surface, SII and PV, that have an internal organization similar to that of other species (Fig. 13). While homologous fields need not be functionally analogous, current results and results in other mammals suggest that certain areas of the cortex are highly conserved in evolution, and are components of a basic processing network that can be identified in most lineages.

\section{References}

Alloway K, Fabri M, Burton H (1990) Ipsilateral corticocortical connections of SI subdivisions in monkeys. Soc Neurosci Abstr 16:228.

Andersen RA, Asanuma C, Essick G, Siegel RM (1990) Corticocortical connections of anatomically and physiologically defined subdivisions within the inferior parietal lobule. J Comp Neurol 296:65-113.

Burton H, Carlson M (1986) Second somatic sensory cortical area (SII) in a prosimian primate, Galago crassicaudatus. J Comp Neurol 247: $200-220$.

Burton H, Robinson CJ (1981) Organization of the SII parietal cortex-multiple somatic sensory representations within and near the second somatic sensory area of cynomolgus monkeys. In: Cortical sensory organization (Carlson M, Welt C, eds), pp 67-119. Clifton, NJ: Humana.

Burton H, Robinson CJ (1987) Responses in the first or second somatosensory cortical area in cats during transient inactivation of the other ipsilateral area with lidocaine hydrochloride. Somatosens Res $4: 215-236$.

Burton H, Alloway KD, Rosenthal P (1988) Somatotopic organization of the second somatosensory cortical area after lesions of the primary somatosensory area in infant and adult cats. Brain Res 448:397-402.

Burton H, Sathian K, Dian-Hua S (1990) Altered responses to cutaneous stimuli in the second somatosensory cortex following lesions of the postcentral gyrus in infant and juvenile macaques. J Comp Neurol 291:395-414.

Burton H, Videen TO, Raichle ME (1993) Tactile-vibration-activated foci in insular and parietal opercular cortex with positron emission tomography: mapping the second somatosensory area in humans. Somatosens Motor Res 10:297-308.

Carlson M, Nystrom P (1994) Tactile discrimination capacity in relation to size and organization of somatic sensory cortex in primates. I. Old-World prosimian, Galago, II. New-World anthropoids, Saimiri and Cebus. J Neurosci 14:1516-1541.

Carroll EW, Wong-Riley MTT (1984) Quantitative light and electron microscopic analysis of cytochrome oxidase-rich zones in the striate cortex of the squirrel monkey. J Comp Neurol 222:1-17.

Clemo HR, Stein BE (1982) Somatosensory cortex: a 'new' somatotopic representation. Brain Res 235:162-168.

Clcmo HR, Stein BE (1983) Organization of a fourth somatosensory area of cortex in cat. J Neurophysiol 50:910-925.

Cusick CG, Wall JT, Felleman DJ, Kaas JH (1989) Somatotopic organization of the lateral sulcus of owl monkeys: area 3b, S-II, and a ventral somatosensory area. J Comp Neurol 282:169-190.

Darian-Smith I, Isbister J, Mok H, Yokota T (1966) Somatic sensory cortical projection areas excited by tactile stimulation of the cat: a triple representation. J Physiol (Lond) 182:671-689.

Dreyer DA, Loe PR, Metz CB, Whitsel BL (1975) Representation of head and face in postcentral gyrus of the macaque. $J$ Neurophysiol 38:714-733

Fabri M, Burton H (1991) Ipsilateral cortical connections of primary somatic sensory cortex in rats. J Comp Neurol 311:405-424.

Feldman SH, Johnson JI (1988) Kinesthetic cortical area anterior to primary somatic sensory cortex in the raccoon (Procyon lotor). $\mathrm{J}$ Comp Neurol 277:80-95.

Friedman DP, Jones EG, Burton H (1980) Representation pattern in the second somatic sensory area of the monkey cerebral cortex. $J$ Comp Neurol 192:21-41.

Friedman DP, Murray EA, O’Neill JB, Mishkin M (1986) Cortical connections of the somatosensory fields of the lateral sulcus of macaques: evidence for a corticolimbic pathway for touch. J Comp Neurol 252:323-347.

Gallyas F (1979) Silver staining of myelin by means of physical development. Neurology 1:203-209.

Garraghty PE, Pons TP, Kaas JH (1990) Ablations of areas 3b (SI proper) and $3 \mathrm{a}$ of somatosensory cortex in marmosets deactivate the second and parietal ventral somatosensory areas. Somatosens Motor Res 7:125-135.

Garraghty PE, Florence SL, Tenhula WN, Kaas J H (1991) Parallel thalamic activation of the first and second somatosensory areas in prosimian primates and tree shrews. J Comp Neurol 311:289-299.

Herron P (1978) Somatotopic organization of mechanosensory projections to SII cerebral neocortex in the raccoon (Procyon lotor). $\mathrm{J}$ Comp Neurol 181:717-728.

Johnson JI (1990) Comparative development of somatic sensory cortex. In: Cercbral cortex (Jones EG, Petcrs A, eds), pp 335-449. New York: Plenum.

Johnson JI, Ostapoff E-M, Warach S (1982) The anterior border zones of primary somatic sensory (SI) neocortex and their relation to cerebral convolutions, shown by micromapping of peripheral projections to the region of the fourth forepaw digit representation in raccoons. Neuroscience 7:915-936.

Jones EG, Porter R (1980) What is area 3a? Brain Res Rev 2:1-43. 
Kaas JH (1983) What, if anything, is SI? Organization of first somatosensory area of cortex. Physiol Rev 63:206-230.

Kaas JH, Pons TP (1988) The somatosensory system of primates. In: Comparative primate biology (Steklis HP, ed), pp 421-468. New York: Liss.

Kaas JH, Nelson RJ, Sur M, Lin C-S, Merzenich MM (1979) Multiple representations of the body within the primary somatosensory cortex of primates. Science 204:521-523.

Krubitzer LA, Calford MB (1992) Five topographically organized fields in the somatosensory cortex of the flying fox: microelectrode maps, myeloarchitecture, and cortical modules. J Comp Neurol 317: $1-30$.

Krubitzer LA, Kaas JH (1990) The organization and connections of somatosensory cortex in marmosets. J Neurosci 10:952-974.

Krubitzer LA, Sesma MA, Kaas JH (1986) Microelectrode maps, myeloarchitecture, and cortical connections of three somatotopically organized representations of the body surface in the parietal cortex of squirrels. J Comp Neurol 250:403-430.

Krubitzer LA, Calford MB, Schmid LM (1993) Connections of somatosensory cortex in megachiropteran bats: the evolution of cortical fields in mammals. J Comp Neurol 327:473-506.

LeClerc SS, Rice FL, Dykes RW, Pourmoghadam K, Gomez CM (1993) Electrophysiological examination of the representation of the face in the suprasylvian gyrus of the ferret: a correlative study with cytoarchitecture. Somatosens Motor Res 10:133-159.

Lund JS, Yoshioka T, Levitt JB (1993) Comparison of intrinsic connectivity in different areas of macaque monkey cerebral cortex. Cereb Cortex 3:148-162.

Manzoni T, Caminiti R, Spidalieri G, Morelli E (1979) Anatomical and functional aspects of the associative projections from somatic area SI to SII. Exp Brain Res 34:453-470.

Merzenich MM, Kaas JH, Sur M, Lin C-S (1978) Double representation of the body surface within cytoarchitectonic areas $3 \mathrm{~b}$ and 1 in "SI" in the owl monkey (Aotus trivirgatus). J Comp Neurol 181:4174.

Neal JW, Pearson RCA, Powell TPS (1990) The ipsilateral corticocortical connections of $7 \mathrm{~b}, \mathrm{PF}$ in the parietal and temporal lobes of the monkey. Brain Res 524:119-132.

Nelson RJ, Sur M, Felleman DJ, Kaas JH (1980) Representations of the body surface in postcentral parietal cortex of Macaca fascicularis. J Comp Neurol 192:61 1-643.

Pons TP, Garraghty PE, Cusick CG, Kaas JH (1985) The somatotopic organization of area 2 in macaque monkeys. J Comp Neurol 241: $445-466$.

Pons TP, Garraghty PE, Mishkin M (1988) Lesion-induced plasticity in the second somatosensory cortex of adult macaques. Proc Nat Acad Sci USA 85:5279-5281.

Robinson CJ, Burton H (1980) Somatotopographic organization in the second somatosensory area of M. fascicularis. J Comp Neurol 192: $43-67$.

Schneider RJ, Friedman DP, Mishkin M (1993) A modality-specific somatosensory area within the insula of the rhesus monkey. Brain Res 621:116-120.

Sur M, Nelson RJ, Kaas JH (1982) Representations of the body surface in cortical areas $3 \mathrm{~b}$ and 1 of squirrel monkeys: comparisons with other primates. J Comp Neurol 211:177-192.

Turman AB, Ferrington DG, Ghosh S, Morley JW, Rowe MJ (1992) Parallel processing of tactile information in the cerebral cortex of the cat: effect of reversible inactivation of SI on responsiveness to SII neurons. J Neurophysiol 647:411-428.

Van Essen DC, Maunsell JHR (1980) Two-dimensional maps of the cerebral cortex. J Comp Neurol 191:255-281.

Whitsel BL, Pertrucelli LM, Werner G (1969) Symmetry and connectivity in the map of the body surface in somatosensory area II of primates. J Neurophysiol 32:170-183.

Wiesendanger M, Miles TS (1982) Ascending pathway of low-threshold muscle afferents to the cerebral cortex and its possible role in motor control. Physiol Rev 62:1223-1270.

Woolsey CN (1958) Organization of somatic sensory and motor areas of the cerebral cortex. In: The biological and biochemical bases of behavior (Harlow HF, Woolsey CN, eds), pp 63-81. Madison: University of Wisconsin.

Woolsey CN, Fairman D (1946) Contralateral, ipsilateral, and bilateral representation of cutancous receptors in somatic arcas I and II of the cerebral cortex of pig, sheep, and other mammals. Surg 19:684-702.

Zhang HQ, Murray GM, Turman AB, Mackie PD, Rowe MJ (1993) Are there direct and indirect paths for tactile inputs to SII from the thalamus in the primate? Proc Aust Neurosci Soc 4:83. 